\title{
Pre-Equalization for MISO DS-UWB Systems with Pre-Rake Combining
}

\author{
Elham Torabi, Student Member, IEEE, Jan Mietzner, Member, IEEE, \\ and Robert Schober, Senior Member, IEEE
}

\begin{abstract}
In this paper, we propose two novel pre-equalization schemes for multiple-input single-output (MISO) directsequence ultra-wideband (DS-UWB) systems with pre-Rake combining and symbol-by-symbol detection. The first scheme employs one pre-equalization filter (PEF) per transmit antenna, whereas in the second scheme, the simplified PEF (S-PEF) scheme, all transmit antennas share the same PEF. For both schemes the optimum finite impulse response (FIR) and infinite impulse response (IIR) PEFs are calculated based on the minimum mean squared error (MMSE) criterion. Our approach is sufficiently general to include also reduced-complexity versions of pre-Rake combining that employ a limited number of Rake fingers. We show that under certain conditions the S-PEF scheme achieves the same performance as the more complex PEF scheme. We also demonstrate that a single-input multipleoutput (SIMO) DS-UWB system with post-Rake combining and MMSE post-equalization is the dual system to the considered MISO DS-UWB system with pre-Rake combining and MMSE pre-equalization. This uplink-downlink duality can be exploited for efficient calculation of the PEFs and for complexity reduction. Our simulation results show that the proposed PEF schemes achieve significant performance gains over pre-Rake combining without equalization, even if only short PEFs are employed.
\end{abstract}

Index Terms-Ultra-wideband systems, spread-spectrum communications, multipath channels, equalization, Rake combining, pre-filtering, multiple-input single-output systems.

\section{INTRODUCTION}

$\mathbf{I}$ N RECENT years, ultra-wideband (UWB) signaling has emerged as a promising solution to high-rate short-range wireless personal area networks. Due to their extremely large bandwidths, UWB systems can resolve even dense multipath components such that Rake combining can be used at the receiver to significantly reduce the negative effects of fading in the received signal [1], [2]. However, for many UWB applications the receiver is a portable device with severely limited signal processing capabilities, making the implementation of Rake combiners with a sufficiently large number of fingers very challenging.

Manuscript received August 26, 2007; revised October 19, 2007 and December 4, 2007; accepted January 29, 2008. The associate editor coordinating the review of this paper and approving it for publication was N. Arumugam.

This work was partly supported by a postdoctoral fellowship from the German Academic Exchange Service (DAAD). Part of this work was presented at the IEEE International Conference on Communications (ICC), Beijing, China, May 2008.

The authors are with the Department of Electrical \& Computer Engineering, The University of British Columbia, 2332 Main Mall, Vancouver, BC, Canada, V6T 1Z4 (e-mail: \{elhamt, janm, rschober\}@ece.ubc.ca).

Digital Object Identifier 10.1109/TWC.2009.070947
A promising approach to overcome this problem is to move computational complexity from the receiver to the more powerful transmitter (e.g. an access point). For this purpose the concept of pre-Rake combining (also referred to as timereversal signaling) was borrowed from other areas such as time-division duplex code-division multiple access (TDDCDMA) systems [3] and underwater acoustic communication [4], and was modified for UWB applications, cf. e.g. [5][13]. Pre-Rake combining exploits the reciprocity of the UWB radio channel, which was recently experimentally confirmed in [11]. Ideally, with pre-Rake combining channel estimation, diversity combining, and equalization are avoided at the receiver, and a simple symbol-by-symbol detector can be used [6], [12]. In addition, it has been recently shown that pre-Rake combining also performs well in the presence of multiple users [9], and the extension to multiple-input single-output (MISO) scenarios was proposed in [9], [11].

Despite all of these desirable properties, pre-Rake combining has a serious drawback. In particular, for the long channel impulse responses (CIRs), which are typical for UWB applications, it may entail a relatively high error floor if simple symbol-by-symbol detection is applied at the receiver [5], [7]. To remedy this problem receiver-side equalization [5], [14] and post-Rake combining [10] have been proposed. However, these techniques increase receiver complexity and thus, compromise to some extend the advantages of preRake combining. Therefore, transmitter-side approaches for performance improvement seem to be more suitable for preRake UWB systems. One option in this regard is to decrease the data rate (i.e., increase the chip or/and symbol duration), which effectively decreases the residual intersymbol interference (ISI) at the receiver [12]. However, if high data rates are desired, some form of pre-equalization has to be applied at the transmitter. In [15] the pre-Rake filter is replaced by a prefilter which minimizes the residual ISI at the receiver based on the minimum mean squared error (MMSE) criterion. Since this MMSE pre-filter is implemented at the chip level, depending on the underlying channel, relatively long filters may be necessary to achieve a good performance. This entails a high complexity, since the computation of the filter coefficients requires the inversion of a matrix with a size equal to the filter length.

In this paper, we propose a novel pre-equalization filter (PEF) scheme for MISO direct-sequence (DS) UWB systems which consists of a bank of pre-Rake filters and a bank of PEFs. Unlike [15], we retain the pre-Rake filters, as they 
efficiently shorten the overall CIRs, and implement the PEFs at the symbol level. As a result, the PEF lengths required to achieve a certain performance are much smaller for the proposed scheme than for the scheme in [15]. ${ }^{1}$ Although preequalization problems have been extensively studied in the literature, e.g. [16], [17], existing results cannot be easily adopted for the problem at hand due to the presence of the pre-Rake, the imposed simple receiver processing, and the spreading applied in DS-UWB. Consequently, in this paper we derive the optimum finite impulse response (FIR) and infinite impulse response (IIR) MMSE PEFs and analyze the performance of the resulting system. Furthermore, we show that a single-input multiple-output (SIMO) system with post-Rake combining followed by MMSE equalization is the dual system for the proposed MISO system with preRake combining and pre-equalization. This uplink-downlink duality [18] can be conveniently exploited for efficient adaptive computation of the PEFs. Furthermore, we also study a simplified PEF (S-PEF) scheme for the MISO system, where the bank of PEFs is replaced by a single PEF. We demonstrate that, under certain conditions, the S-PEF scheme can achieve the same performance as the more complex PEF scheme. Our simulation results confirm that the proposed PEF schemes achieve significant performance gains over pre-Rake structures without equalization and that the performance of IIR PEFs can be closely approached by relatively short FIR PEFs.

Organization: In Section II, we present the considered system and channel model. The proposed PEF scheme is optimized and analyzed in Section III. The uplink-downlink duality and the S-PEF scheme are investigated in Sections IV and V, respectively. In Section VI, simulation results are provided, and Section VII concludes this paper.

Notation: $\mathcal{E}\{\cdot\},[\cdot]^{T},(\cdot)^{*},[\cdot]^{H}$, and $\operatorname{diag}\{\cdot\}$ denote statistical expectation, transposition, complex conjugation, Hermitian transposition, and a (block) diagonal matrix, respectively. $\boldsymbol{I}_{X}$, $\mathbf{0}_{X}, e_{n}, \Re\{\cdot\}$, and $*$ stand for the $X \times X$ identity matrix, the $X$-dimensional all-zeros column vector, the unit vector whose elements are all zero except for the $n$th element which is equal to 1 , the real part of a complex number, and linear convolution, respectively. Furthermore, $Q(x) \triangleq \frac{1}{\sqrt{2 \pi}} \int_{x}^{\infty} e^{-t^{2} / 2} \mathrm{~d} t$, $\delta(\cdot)$, and $X\left(e^{j \omega}\right) \triangleq \mathcal{F}\{x[k]\}=\sum_{k=-\infty}^{\infty} x[k] e^{-j \omega k}$ denote the Gaussian $Q$-function, the Dirac delta function, and the discrete-time Fourier transform of $x[k]$, respectively. Finally, depending on the context, $x[k]$ represents either a sequence or the $k$ th element of a sequence.

\section{System And Channel Model}

We consider a MISO DS-UWB system with $M$ perfectly synchronized transmit antennas, symbol duration $T_{s}$, and chip duration $T_{c}=T_{s} / N$, where $N$ is the spreading factor. A block diagram of the discrete-time model of this system is

\footnotetext{
${ }^{1}$ In both schemes the complexity of the pre-filter computation typically scales with the cube of the filter length, whereas the computation of the additional pre-Rake filter required for the proposed transmitter structure scales only linearly with the number of Rake fingers. Therefore, it appears to be fair to say that the proposed transmitter structure requires a smaller overall computational complexity, especially with regard to the long CIRs encountered in typical UWB applications.
}

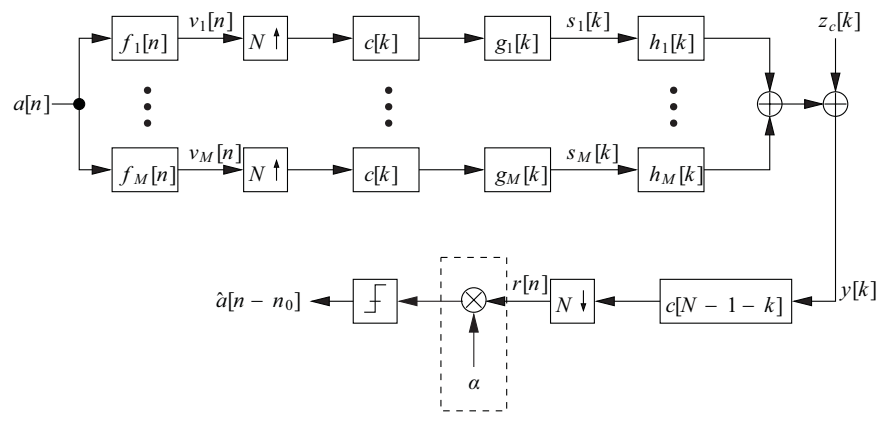

Fig. 1. Block diagram of MISO DS-UWB system (downlink) with $M$ transmit antennas, pre-Rake combining, and pre-equalization. The multiplication of $r[n]$ with $\alpha$ (dashed box) does not have to be implemented at the receiver, cf. discussion in Sections II-C and III.

shown in Fig. 1. For simplicity, we focus on the single-user case throughout this paper. We note that our results could be extended to multiple receive antennas in a straightforward fashion. However, for the sake of clarity and since a simple receiver structure is desired, we assume that only a single receive antenna is available. To emphasize the different capabilities of the transmitter and the receiver we will also refer to the system in Fig. 1 as the downlink. Furthermore, for convenience, all signals and systems are represented by their complex baseband equivalents. In the following, the transmitter structure of the proposed PEF scheme, the adopted channel model, and the receiver structure are discussed.

\section{A. Transmitter Structure}

At antenna $m, 1 \leq m \leq M$, the transmitted independent and identically distributed (i.i.d.) data symbols $a[n] \in\{ \pm 1\}$ are filtered with PEF $f_{m}[n]$ of length $L_{f}$, and the filter output signal

$$
v_{m}[n] \triangleq f_{m}[n] * a[n]=\sum_{l=0}^{L_{f}-1} f_{m}[l] a[n-l]
$$

is up-sampled by a factor of $N$. The up-sampled signal is then filtered with (real-valued) spreading sequence $c[k]$, $0 \leq k<N$, and with pre-Rake filter $g_{m}[k]$ of length $L_{g}$. For convenience the spreading sequence is normalized to $\sum_{k=0}^{N-1}|c[k]|^{2}=1$. The resulting transmit symbol $s_{m}[k]$ is given by

$$
s_{m}[k]=\sum_{i=-\infty}^{\infty} v_{m}[i] \tilde{g}_{m}[k-i N],
$$

where $\tilde{g}_{m}[k] \triangleq c[k] * g_{m}[k]=\sum_{i=0}^{N-1} c[i] g_{m}[k-i]$ includes the combined effects of the pre-Rake filter and spreading. We note that the considered transmitter structure is very general as we do not impose any restrictions on $c[k]$ and $g_{m}[k]$. If a spreading sequence is not applied, e.g. [5], [9], [11], [15], we have $c[0]=1$ and $c[k]=0,1 \leq k<N$. In general, $g_{m}[k]$, $1 \leq m \leq M$, will depend in some way on the CIR $h_{m}[k]$, which has length $L_{h}$. For example, an all-pre-Rake (A-preRake or time-reversal filter)

$$
g_{m}[k] \triangleq h_{m}^{*}\left[L_{h}-k-1\right], 0 \leq k<L_{g}, L_{g}=L_{h},
$$

a partial-pre-Rake (P-pre-Rake)

$$
g_{m}[k] \triangleq h_{m}^{*}\left[L_{g}-k-1\right], 0 \leq k<L_{g}, L_{g}<L_{h},
$$


or a selective pre-Rake (S-pre-Rake) $g_{m}[k] \triangleq h_{m}^{*}\left[L_{g}-k-1\right]$ for the $S$ largest coefficients of $h_{m}[k], g_{m}[k] \triangleq 0$ otherwise, and $L_{g} \leq L_{h}$ may be adopted.

Since for typical UWB CIR lengths it is not realistic to assume that the CIR coefficients can be fed back from the receiver to the transmitter [13], as any UWB transmitter structure requiring channel state information, the proposed PEF scheme hinges on the reciprocity of the UWB channel. Fortunately, this reciprocity has been experimentally confirmed [11]. Hence, $h_{m}[k]$ can be estimated at the transmitter, relieving the receiver from any channel estimation tasks.

\section{B. Channel Model}

The equivalent baseband discrete-time CIRs

$$
\left.h_{m}[k] \triangleq g_{T}(t) * h_{m}(t) * g_{R}(t)\right|_{k T_{c}},
$$

$1 \leq m \leq M$, contain the combined effects of the transmit filter $g_{T}(t)$, the continuous-time CIR $h_{m}(t)$, and the receive filter $g_{R}(t) .^{2}$ Furthermore, for the wireless channel we adopt the recently proposed extension of the IEEE 802.15.3a channel model [19], [20] to multiple antennas [21]. Consequently, the passband version $h_{m}^{\prime}(t)$ of the baseband CIR $h_{m}(t)$ consists of $L_{c}$ clusters of $L_{r}$ rays and is modeled as

$$
h_{m}^{\prime}(t)=X_{m} \sum_{l=1}^{L_{c}} \sum_{k=1}^{L_{r}} \alpha_{k, l, m} \delta\left(t-T_{l, m}-\tau_{k, l, m}\right),
$$

where $T_{l, m}$ is the delay of the $l$ th cluster, $\tau_{k, l, m}$ is the delay of the $k$ th ray of the $l$ th cluster, $\alpha_{k, l, m}$ is the random multipath gain coefficient, and $X_{m}$ models the lognormal shadowing. ${ }^{3}$ Measurements reported in [21] have confirmed that while $T_{l, m}, \tau_{k, l, m}$, and $\alpha_{k, l, m}$ are independent across antennas, the lognormal terms $X_{m}$ are mutually correlated. In [19], [20] four parameter sets for the various channel model parameters in Eq. (3) are specified. The resulting four channel models (CMs) are known as CM1, CM2, CM3, and CM4. They represent different usage scenarios and entail different amounts of ISI.

\section{Receiver Structure}

The received signal $y[k]$ is filtered with the time-reversed spreading sequence $c[N-1-k], 0 \leq k<N$, and sampled at times $k=N n+k_{0}$, where $0 \leq k_{0}<N$ denotes the sampling phase. The resulting receiver output signal $r[n]$ can be expressed as

$$
r[n]=\sum_{m=1}^{M} \sum_{l=-\infty}^{\infty} q_{m}\left[N l+k_{0}\right] v_{m}[n-l]+z_{s}[n],
$$

with overall CIR

$$
q_{m}[k] \triangleq \tilde{g}_{m}[k] * \tilde{h}_{m}[k]=\sum_{i=0}^{L_{g}+N-2} \tilde{g}_{m}[i] \tilde{h}_{m}[k-i],
$$

\footnotetext{
${ }^{2}$ Note that the continuous-time overall CIR $g_{T}(t) * h_{m}(t) * g_{R}(t)$ has infinite length. However, the discrete-time version $h_{m}[k]$ can be truncated to a finite length $L_{h}$ with negligible energy loss if $L_{h}$ is chosen sufficiently large, cf. Section VI.

${ }^{3}$ We assume that the signals transmitted over different antennas are time synchronous as is customary in the literature, cf. e.g. [5]-[15]. The delays $T_{l, m}$ and $\tau_{k, l, m}$ in Eq. (3) only specify the exact location of individual CIR taps due to reflections from scatterers within the environment.
}

$\tilde{h}_{m}[k] \triangleq h_{m}[k] * c[N-1-k]=\sum_{i=0}^{N-1} c[i] h_{m}[k+i-(N-1)]$, and symbol-level noise

$$
z_{s}[n]=\sum_{i=0}^{N-1} c[i] z_{c}\left[N(n-1)+k_{0}+i+1\right],
$$

where $z_{c}[k]$ denotes the chip-level additive white Gaussian noise (AWGN) with variance $\sigma_{c}^{2} \triangleq \mathcal{E}\left\{\left|z_{c}[k]\right|^{2}\right\}$. Consequently, $z_{s}[n]$ is also AWGN with variance $\sigma_{s}^{2} \triangleq \mathcal{E}\left\{\left|z_{s}[n]\right|^{2}\right\}=$ $\sigma_{c}^{2} \sum_{i=0}^{N-1}|c[i]|^{2}=\sigma_{c}^{2}$. Ideally, the sampling phase $k_{0}$ should be optimized to maximize the energy of $q_{m}\left[N l+k_{0}\right]$. However, the number of operations required for finding the optimum $k_{0}$ may be prohibitive, especially for large $N$. Therefore, in practice, it may be preferable to consider suboptimum choices for $k_{0}$ which yield a good performance and can be easily found. For an A-pre-Rake combiner $q_{m}[k]$ will assume its maximum for $k=L_{g}+N-2$. In order to capture this maximum in $q_{m}\left[N l+k_{0}\right]$, we require

$$
k_{0}=k_{0}^{s} \triangleq L_{g}+N-2-N\left\lfloor\left(L_{g}+N-2\right) / N\right\rfloor .
$$

Through extensive simulations we found that $k_{0}^{s}$ yields a close-to-optimum performance not only for A-pre-Rake combining but also for $\mathrm{S}$ - and $\mathrm{P}$-pre-Rake combining as long as the number of fingers is sufficiently large. ${ }^{4}$

Since the goal of the proposed UWB system design is to minimize receiver complexity in the downlink, no additional filtering is applied at the receiver and symbol decisions are made according to ${ }^{5}$

$$
\hat{a}\left[n-n_{0}\right]=\operatorname{sign}\{\Re\{r[n]\}\},
$$

where $\hat{a}\left[n-n_{0}\right]$ is the estimate for $a\left[n-n_{0}\right], n_{0}$ denotes the decision delay, and $\operatorname{sign}\{x\}=1$ if $x \geq 0$ and $\operatorname{sign}\{x\}=-1$ otherwise. As typical for equalization problems, the decision delay $n_{0}$ has to be optimized for performance maximization if causal pre-filters are desired [22]. ${ }^{6}$

\section{PEF Optimization AND PERFormance ANALYSiS}

In this paper, we adopt the MMSE criterion for optimization of the PEFs $f_{m}[n]$. In particular, our design goal is to minimize the error variance

$$
\sigma_{e}^{2} \triangleq \mathcal{E}\left\{\left|a\left[n-n_{0}\right]-\alpha r[n]\right|^{2}\right\},
$$

while limiting the power $P$ of the transmitted signal over one symbol interval, i.e.,

$$
P \triangleq \sum_{k=N n}^{N(n+1)-1} \sum_{m=1}^{M} \mathcal{E}\left\{\left|s_{m}[k]\right|^{2}\right\}=1, \quad \forall n .
$$

\footnotetext{
${ }^{4}$ To be specific, for all simulation results presented in the paper, the performance resulting for $k_{0}=k_{0}^{s}$ was virtually indistinguishable from that resulting for the optimum choice of $k_{0}$, while the number of Rake fingers considered was as low as $S=8$. We have therefore adopted $k_{0}=k_{0}^{s}$ for all our simulation results in Section VI.

${ }^{5}$ Note that the multiplication with $\alpha$ in Fig. 1 does not have to be implemented at the receiver, see discussion in Section III.

${ }^{6}$ Typically, the decision delay $n_{0}$ is chosen such that the number of precursor taps within the resulting overall CIR is (roughly) equal to the number of post-cursor taps. The required complexity for optimizing the decision delay $n_{0}$ is common to all FIR pre-equalization schemes considered in this paper.
} 
Here, $\alpha$ in Eq. (6) is an auxiliary variable that simplifies the optimization of the PEFs $f_{m}[n]$ but does not affect the symbol decisions according to Eq. (5).

In the following subsections, we will derive the optimum FIR and IIR PEFs based on Eqs. (6) and (7) and analyze their performance.

\section{A. FIR Pre-Equalization Filters}

For FIR PEF optimization it is convenient to first rewrite Eq. (4) as

$$
r[n]=\sum_{m=1}^{M}\left(\boldsymbol{Q}_{m} \boldsymbol{f}_{m}\right)^{H} \boldsymbol{a}[n]+z_{s}[n]=(\boldsymbol{Q} \boldsymbol{f})^{H} \boldsymbol{a}[n]+z_{s}[n],
$$

where $\boldsymbol{a}[n] \triangleq\left[a[n] \ldots a\left[n-L_{t}+1\right]\right]^{T}, \boldsymbol{f} \triangleq\left[\boldsymbol{f}_{1}^{T} \ldots \boldsymbol{f}_{M}^{T}\right]^{T}$, $\boldsymbol{f}_{m} \triangleq\left[f_{m}[0] \ldots f_{m}\left[L_{f}-1\right]\right]^{H}, \boldsymbol{Q} \triangleq\left[\boldsymbol{Q}_{1} \ldots \boldsymbol{Q}_{M}\right]$, and $\boldsymbol{Q}_{m}$ denotes an $L_{t} \times L_{f}$ column-circulant matrix with vector

$$
\left[q_{m}\left[k_{0}\right] q_{m}\left[N+k_{0}\right] \ldots q_{m}\left[N\left(L_{q}-1\right)+k_{0}\right] \mathbf{0}_{L_{f}-1}^{T}\right]^{H}
$$

as first column. Here,

$$
L_{t} \triangleq L_{q}+L_{f}-1
$$

and

$$
L_{q}=\left\lceil\left(L_{g}+L_{h}+2 N-3\right) / N\right\rceil
$$

are the lengths of the impulse response of the overall system (including the PEFs) and the sampled overall CIRs $q_{m}\left[N n+k_{0}\right], 1 \leq m \leq M$, respectively. ${ }^{7}$ Applying Eq. (8) in Eq. (6) yields

$$
\sigma_{e}^{2}=1+|\alpha|^{2} \sigma_{c}^{2}-\alpha \boldsymbol{f}^{H} \boldsymbol{q}-\alpha^{*} \boldsymbol{q}^{H} \boldsymbol{f}+|\alpha|^{2} \boldsymbol{f}^{H} \boldsymbol{Q}^{H} \boldsymbol{Q} \boldsymbol{f},
$$

where $\boldsymbol{q} \triangleq \boldsymbol{Q}^{H} \boldsymbol{e}_{n_{0}}$. Furthermore, we show in Appendix A that the average transmit power $P$ can be expressed as

$$
P=\boldsymbol{f}^{H} \boldsymbol{\Phi} \boldsymbol{f}
$$

with $M L_{f} \times M L_{f}$ block diagonal matrix

$$
\boldsymbol{\Phi} \triangleq \operatorname{diag}\left\{\boldsymbol{\Phi}_{1}, \ldots, \boldsymbol{\Phi}_{M}\right\} .
$$

Here, $\boldsymbol{\Phi}_{m}$ is a symmetric Toeplitz matrix with vector

$$
\left[\varphi_{m}[0] \varphi_{m}[-N] \ldots \varphi_{m}\left[-N\left(L_{f}-1\right)\right]\right]
$$

in its first row, where $\varphi_{m}[k] \triangleq \tilde{g}_{m}[k] * \tilde{g}_{m}^{*}[-k]$. Combining Eqs. (9) and (10) we obtain the Lagrange problem

$$
\begin{aligned}
L(\boldsymbol{f}, \alpha)= & 1+|\alpha|^{2} \sigma_{c}^{2}-\alpha \boldsymbol{f}^{H} \boldsymbol{q}-\alpha^{*} \boldsymbol{q}^{H} \boldsymbol{f} \\
& +|\alpha|^{2} \boldsymbol{f}^{H} \boldsymbol{Q}^{H} \boldsymbol{Q} \boldsymbol{f}+\lambda\left(\boldsymbol{f}^{H} \boldsymbol{\Phi} \boldsymbol{f}-1\right),
\end{aligned}
$$

where $\lambda$ denotes the Lagrange multiplier. Differentiating $L(\boldsymbol{f}, \alpha)$ with respect to $\boldsymbol{f}^{*}$ and $\alpha^{*}$ and setting the resulting gradients to zero leads to the optimum solution

$$
\boldsymbol{f}_{\mathrm{opt}}=\frac{1}{\alpha_{\mathrm{opt}}^{*}}\left(\boldsymbol{Q}^{H} \boldsymbol{Q}+\sigma_{c}^{2} \boldsymbol{\Phi}\right)^{-1} \boldsymbol{q},
$$

\footnotetext{
${ }^{7}$ Note that $q_{m}[k]$ has length $L_{g}+L_{h}+2 N-3$ as it is the result of the convolution of $g_{m}[k]$ (length: $L_{g}$ ), $h_{m}[k]$, (length: $L_{h}$ ), $c[k]$ (length: $N$ ), and $c[N-1-k]$ (length: $N)$. The length of $q_{m}\left[N n+k_{0}\right]$ is then obtained as $L_{q}=\left\lceil\left(L_{g}+L_{h}+2 N-3\right) / N\right\rceil$ by taking the effect of the sampling into account.
}

$$
\alpha_{\mathrm{opt}}=\sqrt{\boldsymbol{q}^{H}\left(\boldsymbol{Q}^{H} \boldsymbol{Q}+\sigma_{c}^{2} \boldsymbol{\Phi}\right)^{-1} \boldsymbol{\Phi}\left(\boldsymbol{Q}^{H} \boldsymbol{Q}+\sigma_{c}^{2} \boldsymbol{\Phi}\right)^{-1} \boldsymbol{q}}
$$

We note that the error variance does not change if we multiply the right hand side of Eq. (13) with $e^{j \phi}$, where $\phi$ is an arbitrary phase. However, from a practical point of view real-valued positive values for $\alpha_{\mathrm{opt}}$ are desirable, since in that case $\alpha_{\mathrm{opt}}$ does not have to be accounted for in the symbol decision rule at the receiver, cf. Eq. (5). Using Eqs. (12) and (13) in Eq. (9) leads to the minimum error variance ${ }^{8}$

$$
\sigma_{e, \min }^{2}=1-\boldsymbol{q}^{H}\left(\boldsymbol{Q}^{H} \boldsymbol{Q}+\sigma_{c}^{2} \boldsymbol{\Phi}\right)^{-1} \boldsymbol{q} .
$$

For calculation of $\boldsymbol{f}_{\mathrm{opt}}$ an $M L_{f} \times M L_{f}$ matrix has to be inverted, which is computationally expensive for large filter lengths $L_{f}$. Therefore, from a complexity point of view short FIR filters are desirable. On the other hand, the performance of the proposed pre-equalization scheme improves with increasing $L_{f}$. Therefore, we are interested in finding the minimum value of $L_{f}$ which achieves close-to-optimum performance. In this context, the optimum IIR solution is useful as it allows us to establish the ultimate performance limit of the proposed PEF scheme.

\section{B. IIR Pre-Equalization Filters}

As customary for IIR filter optimization, we drop the causality constraint, set $n_{0}=0$, and use a frequency-domain approach for filter optimization [23]. The frequency-domain approach simplifies our derivations as it avoids having to deal with infinite-length impulse responses. We denote the vector of IIR PEF frequency responses by

$$
\boldsymbol{F}\left(e^{j \omega}\right) \triangleq\left[F_{1}\left(e^{j \omega}\right) \ldots F_{M}\left(e^{j \omega}\right)\right]^{H},
$$

where $F_{m}\left(e^{j \omega}\right) \triangleq \mathcal{F}\left\{f_{m}[n]\right\}$. Similarly, the vector of the Fourier transforms of the sampled overall CIRs $q_{m}\left[N n+k_{0}\right]$ is defined as

$$
\boldsymbol{Q}\left(e^{j \omega}\right) \triangleq\left[Q_{1}\left(e^{j \omega}\right) \ldots Q_{M}\left(e^{j \omega}\right)\right]^{T},
$$

where $Q_{m}\left(e^{j \omega}\right) \triangleq \mathcal{F}\left\{q_{m}\left[N n+k_{0}\right]\right\}$. Note that $Q_{m}\left(e^{j \omega}\right)$ is related to the Fourier transform $\tilde{Q}_{m}\left(e^{j \omega}\right) \triangleq \mathcal{F}\left\{q_{m}\left[k+k_{0}\right]\right\}$ of the (time-shifted) overall CIR itself via [24, Ch. 7]

$$
Q_{m}\left(e^{j \omega}\right)=\frac{1}{N} \sum_{k=0}^{N-1} \tilde{Q}_{m}\left(e^{j(\omega-2 \pi k) / N}\right) .
$$

We show in Appendix B that with these definitions the error variance in Eq. (9) can be rewritten as

$$
\begin{aligned}
\sigma_{e}^{2}= & 1+|\alpha|^{2} \sigma_{c}^{2}- \\
& \frac{1}{2 \pi} \int_{-\pi}^{\pi}\left[\alpha \boldsymbol{F}^{H}\left(e^{j \omega}\right) \boldsymbol{Q}\left(e^{j \omega}\right)+\alpha^{*} \boldsymbol{Q}^{H}\left(e^{j \omega}\right) \boldsymbol{F}\left(e^{j \omega}\right)\right. \\
& \left.-|\alpha|^{2} \boldsymbol{F}^{H}\left(e^{j \omega}\right) \boldsymbol{Q}\left(e^{j \omega}\right) \boldsymbol{Q}^{H}\left(e^{j \omega}\right) \boldsymbol{F}\left(e^{j \omega}\right)\right] \mathrm{d} \omega .
\end{aligned}
$$

${ }^{8}$ Note that the error variance $\sigma_{e, \min }^{2}$ and thus the performance of the PEF scheme depends on a number of system parameters, such as the CIR length $L_{h}$, the pre-Rake filter length $L_{g}$, and the PEF length $L_{f}$. However, as customary for equalization problems, it is not possible to state an explicit analytical relation between $\sigma_{e, \text { min }}^{2}$ and, say, the CIR length $L_{h}$. In fact, the performance of the PEF scheme is rather dominated by the positions of the poles of the (sampled) overall CIRs $q_{m}\left[N n+k_{0}\right]$. 
Similarly, using the definitions

$$
\boldsymbol{\Phi}\left(e^{j \omega}\right) \triangleq \operatorname{diag}\left\{\Phi_{1}\left(e^{j \omega}\right), \Phi_{2}\left(e^{j \omega}\right), \ldots, \Phi_{M}\left(e^{j \omega}\right)\right\}
$$

and $\Phi_{m}\left(e^{j \omega}\right) \triangleq \mathcal{F}\left\{\varphi_{m}[N n]\right\}$ it is shown in Appendix A that the average transmit power in Eq. (10) can be expressed as

$$
P=\frac{1}{2 \pi} \int_{-\pi}^{\pi} \boldsymbol{F}^{H}\left(e^{j \omega}\right) \boldsymbol{\Phi}\left(e^{j \omega}\right) \boldsymbol{F}\left(e^{j \omega}\right) \mathrm{d} \omega .
$$

We note that the Fourier transform $\Phi_{m}\left(e^{j \omega}\right)$ of the sampled sequence $\varphi_{m}[N n]$ is related to the Fourier transform $\tilde{\Phi}_{m}\left(e^{j \omega}\right) \triangleq \mathcal{F}\left\{\varphi_{m}[k]\right\}$ of the sequence itself by [24, Ch. 7]

$$
\Phi_{m}\left(e^{j \omega}\right)=\frac{1}{N} \sum_{k=0}^{N-1} \tilde{\Phi}_{m}\left(e^{j(\omega-2 \pi k) / N}\right) .
$$

Based on Eqs. (16) and (17) we can now formulate a Lagrange problem similar to Eq. (11) in the FIR case. Solving this Lagrange problem leads to the frequency response of the optimum IIR PEFs

$$
\boldsymbol{F}_{\mathrm{opt}}\left(e^{j \omega}\right)=\frac{1}{\alpha_{\mathrm{opt}}^{*}} \boldsymbol{M}\left(e^{j \omega}\right) \boldsymbol{Q}\left(e^{j \omega}\right)
$$

$\alpha_{\mathrm{opt}}=\sqrt{\frac{1}{2 \pi} \int_{-\pi}^{\pi} \boldsymbol{Q}^{H}\left(e^{j \omega}\right) \boldsymbol{M}^{H}\left(e^{j \omega}\right) \boldsymbol{\Phi}\left(e^{j \omega}\right) \boldsymbol{M}\left(e^{j \omega}\right) \boldsymbol{Q}\left(e^{j \omega}\right) \mathrm{d} \omega}$, with

$$
\boldsymbol{M}\left(e^{j \omega}\right) \triangleq\left(\boldsymbol{Q}\left(e^{j \omega}\right) \boldsymbol{Q}^{H}\left(e^{j \omega}\right)+\sigma_{c}^{2} \boldsymbol{\Phi}\left(e^{j \omega}\right)\right)^{-1} .
$$

Using the matrix inversion lemma [25], we obtain

$$
\begin{aligned}
\boldsymbol{M}\left(e^{j \omega}\right)= & \frac{1}{\sigma_{c}^{2}} \boldsymbol{\Phi}^{-1}\left(e^{j \omega}\right) \\
& \times\left[\boldsymbol{I}_{M}-\frac{\boldsymbol{Q}\left(e^{j \omega}\right) \boldsymbol{Q}^{H}\left(e^{j \omega}\right) \boldsymbol{\Phi}^{-1}\left(e^{j \omega}\right)}{\sigma_{c}^{2}+\boldsymbol{Q}^{H}\left(e^{j \omega}\right) \boldsymbol{\Phi}^{-1}\left(e^{j \omega}\right) \boldsymbol{Q}\left(e^{j \omega}\right)}\right] .
\end{aligned}
$$

With this result Eqs. (18) and (19) can be simplified to

$$
\begin{aligned}
F_{m}^{\mathrm{opt}}\left(e^{j \omega}\right) & =\frac{1}{\alpha_{\mathrm{opt}}^{*}} \frac{Q_{m}\left(e^{j \omega}\right)}{\Phi_{m}\left(e^{j \omega}\right)\left(\sigma_{c}^{2}+X\left(e^{j \omega}\right)\right)} \\
\alpha_{\mathrm{opt}} & =\sqrt{\frac{1}{2 \pi} \int_{-\pi}^{\pi} \frac{X\left(e^{j \omega}\right)}{\left(\sigma_{c}^{2}+X\left(e^{j \omega}\right)\right)^{2}} \mathrm{~d} \omega},
\end{aligned}
$$

where

$$
X\left(e^{j \omega}\right)=\boldsymbol{Q}^{H}\left(e^{j \omega}\right) \boldsymbol{\Phi}^{-1}\left(e^{j \omega}\right) \boldsymbol{Q}\left(e^{j \omega}\right)=\sum_{m=1}^{M} \frac{\left|Q_{m}\left(e^{j \omega}\right)\right|^{2}}{\Phi_{m}\left(e^{j \omega}\right)},
$$

and $F_{m}^{\text {opt }}\left(e^{j \omega}\right), 1 \leq m \leq M$, denotes the $m$ th component of $\boldsymbol{F}_{\text {opt }}\left(e^{j \omega}\right)$. The corresponding minimum error variance can be obtained from Eq. (16) as

$$
\sigma_{e, \min }^{2}=\frac{1}{2 \pi} \int_{-\pi}^{\pi} \frac{\sigma_{c}^{2}}{\sigma_{c}^{2}+X\left(e^{j \omega}\right)} \mathrm{d} \omega .
$$

\section{Performance Analysis}

In this subsection, we provide an analytical approximation for the bit-error rate (BER) of the proposed pre-equalization scheme. For this purpose, we assume that the residual ISI is approximately Gaussian distributed, which is typically a good assumption for MMSE problems [26]. We first assume that FIR PEFs are used and note that the receiver output signal can be expressed as

$$
r[n]=\boldsymbol{f}^{H} \boldsymbol{q} a\left[n-n_{0}\right]+\boldsymbol{f}^{H} \boldsymbol{Q}^{H} \boldsymbol{a}_{n_{0}}[n]+z_{s}[n],
$$

where $\boldsymbol{a}_{n_{0}}[n]$ is identical to $\boldsymbol{a}[n]$ except that its $n_{0}$ th component is zero. Based on this representation, it can be observed that the effective signal-to-noise ratio (SNR) of the decision variable $r[n]$ is given by

$$
\begin{aligned}
\mathrm{SNR} & =\frac{\left|\boldsymbol{f}^{H} \boldsymbol{q}\right|^{2}}{\boldsymbol{f}^{H} \boldsymbol{Q}^{H} \boldsymbol{Q} \boldsymbol{f}-\left|\boldsymbol{f}^{H} \boldsymbol{q}\right|^{2}+\sigma_{c}^{2}} \\
& =\frac{\boldsymbol{q}^{H}\left(\boldsymbol{Q}^{H} \boldsymbol{Q}+\sigma_{c}^{2} \boldsymbol{\Phi}\right)^{-1} \boldsymbol{q}}{1-\boldsymbol{q}^{H}\left(\boldsymbol{Q}^{H} \boldsymbol{Q}+\sigma_{c}^{2} \boldsymbol{\Phi}\right)^{-1} \boldsymbol{q}}=\frac{1}{\sigma_{e, \text { min }}^{2}}-1,
\end{aligned}
$$

where we have used Eqs. (12)-(14). Assuming now that the residual interference term $\boldsymbol{f}^{H} \boldsymbol{Q}^{H} \boldsymbol{a}_{n_{0}}[n]+z_{s}[n]$ is approximately Gaussian distributed, we obtain for the BER of the proposed FIR pre-equalization scheme the approximation

$$
P_{e} \approx Q\left(\sqrt{2\left(\frac{1}{\sigma_{e, \min }^{2}}-1\right)}\right) .
$$

The same expression is also valid for IIR PEFs if the corresponding error variance $\sigma_{e, \text { min }}^{2}$ given in Eq. (22) is used.

\section{Optimality of A-Pre-Rake Combining for IIR PEFs}

It is well known that the performance of pre-Rake (and post-Rake) combiners does not necessarily improve if the number of Rake fingers is increased, cf. e.g. [27]. The reason for this behavior is that while more energy can be collected by increasing the number of fingers, the amount of residual ISI may also increase. A similar effect can be observed if the pre-Rake filter is enhanced with short FIR PEFs. However, we will show in the following that A-pre-Rake combining is indeed optimum if the employed PEFs are sufficiently long. ${ }^{9}$

For this purpose we use Eq. (15) and the corresponding definition of $\Phi_{m}\left(e^{j \omega}\right)$ to rewrite $X\left(e^{j \omega}\right)$ as

$$
\begin{aligned}
& X\left(e^{j \omega}\right)= \\
& \frac{1}{N} \sum_{m=1}^{M} \frac{\left|\sum_{k=0}^{N-1} \tilde{G}_{m}\left(e^{j(\omega-2 \pi k) / N}\right) \tilde{H}_{m}^{\prime}\left(e^{j(\omega-2 \pi k) / N}\right)\right|^{2}}{\sum_{k=0}^{N-1}\left|\tilde{G}_{m}\left(e^{j(\omega-2 \pi k) / N}\right)\right|^{2}}
\end{aligned}
$$

where

$$
\tilde{H}_{m}^{\prime}\left(e^{j(\omega-2 \pi k) / N}\right) \triangleq e^{j(\omega-2 \pi k) k_{0} / N} \tilde{H}_{m}\left(e^{j(\omega-2 \pi k) / N}\right),
$$
$\tilde{G}_{m}\left(e^{j \omega}\right) \triangleq \mathcal{F}\left\{\tilde{g}_{m}[k]\right\}$, and $\tilde{H}_{m}\left(e^{j \omega}\right) \triangleq \mathcal{F}\left\{\tilde{h}_{m}[k]\right\}$. Moreover, we have used that

$$
\tilde{Q}_{m}\left(e^{j \omega}\right)=e^{j \omega k_{0}} \tilde{G}_{m}\left(e^{j \omega}\right) \tilde{H}_{m}\left(e^{j \omega}\right)
$$

${ }^{9}$ As will be seen in Section VI, depending on the channel model under consideration filter lengths of $L_{f}=40$ to $L_{f}=90$ already offer a performance that is virtually indistinguishable from that in the IIR case (cf. Fig. 4). 
and

$$
\tilde{\Phi}_{m}\left(e^{j \omega}\right)=\left|\tilde{G}_{m}\left(e^{j \omega}\right)\right|^{2} .
$$

Using the Cauchy-Schwarz inequality [25] it can be shown that $X\left(e^{j \omega}\right)$ is maximized if

$$
\tilde{G}_{m}\left(e^{j \omega}\right)=e^{-j \omega k_{0}} \tilde{H}_{m}^{*}\left(e^{j \omega}\right)
$$

corresponding to an A-pre-Rake (or time-reversal) filter for each branch $m, 1 \leq m \leq M$. Therefore, the A-pre-Rake filter minimizes the error variance $\sigma_{e, \min }^{2}$ and is optimum if IIR PEFs are employed. The corresponding minimum error variance is given by

$$
\begin{aligned}
& \sigma_{e, \min }^{2}= \\
& \frac{1}{2 \pi} \int_{-\pi}^{\pi} \frac{\sigma_{c}^{2}}{\sigma_{c}^{2}+\frac{1}{N} \sum_{m=1}^{M} \sum_{k=0}^{N-1}\left|\tilde{H}_{m}\left(e^{j(\omega-2 \pi k) / N}\right)\right|^{2}} \mathrm{~d} \omega .
\end{aligned}
$$

Increasing the spreading factor $N$ improves performance by decreasing the effective spectral fluctuation for a given $m$, i.e., $\frac{1}{N} \sum_{k=0}^{N-1}\left|\tilde{H}_{m}\left(e^{j(\omega-2 \pi k) / N}\right)\right|^{2}$ becomes smoother which has a positive effect on $\sigma_{e \text {,min }}^{2}$ in Eq. (26). Increasing the number of antennas has a similar effect, but also provides an additional combining gain. The impact of $N$ on the performance will be investigated in more detail in Section III-F.

\section{E. Performance Bound}

A meaningful performance bound for any (pre-)equalizer and any (pre-)Rake scheme is the so-called matched-filter (MF) bound. For the MF bound we assume that the optimum A-pre-Rake filter is used at the transmitter and ignore any ISI caused at the receiver. The resulting SNR is

$$
\begin{aligned}
\mathrm{SNR}_{\mathrm{MF}} & =\frac{1}{\sigma_{c}^{2}} \sum_{m=1}^{M} \sum_{k=-\infty}^{\infty}\left|\tilde{h}_{m}[k]\right|^{2} \\
& =\frac{1}{2 \pi \sigma_{c}^{2}} \sum_{m=1}^{M} \int_{-\pi}^{\pi}\left|\tilde{H}_{m}\left(e^{j \omega}\right)\right|^{2} \mathrm{~d} \omega,
\end{aligned}
$$

where we have applied Parseval's theorem [24]. The corresponding BER lower bound is

$$
P_{\mathrm{MF}}=Q\left(\sqrt{2 \mathrm{SNR}_{\mathrm{MF}}}\right),
$$

i.e., no implementable (pre-)equalizer and (pre-)Rake scheme will achieve a better performance.

\section{F. Performance for $N \rightarrow \infty$}

It is interesting to investigate the performance of the proposed PEF scheme for the case of long spreading sequences, i.e., $N \rightarrow \infty$. For $N \rightarrow \infty$ the summations over $0 \leq k<N$ in Eq. (25) can be replaced by integrals and $X\left(e^{j \omega}\right)=X$ becomes independent of $\omega$. In particular, we obtain

$$
X=\sum_{m=1}^{M} \frac{\left|\frac{1}{2 \pi} \int_{-\pi}^{\pi} \tilde{G}_{m}\left(e^{j \omega}\right) e^{j \omega k_{0}} \tilde{H}_{m}\left(e^{j \omega}\right) \mathrm{d} \omega\right|^{2}}{\frac{1}{2 \pi} \int_{-\pi}^{\pi}\left|\tilde{G}_{m}\left(e^{j \omega}\right)\right|^{2} \mathrm{~d} \omega},
$$

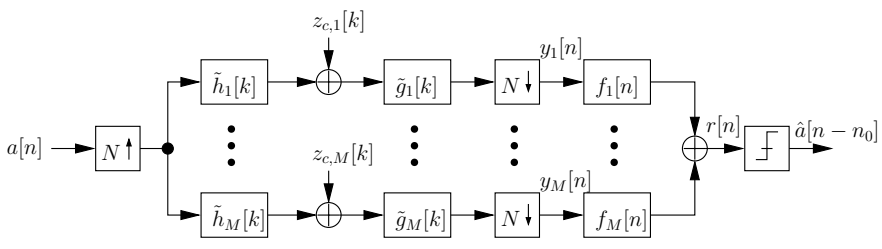

Fig. 2. Block diagram of SIMO DS-UWB system (uplink) with $M$ receive antennas, post-Rake combining, and post-equalization.

and with Eqs. (22) and (23) the resulting effective SNR is $\mathrm{SNR}=X / \sigma_{c}^{2}$. For the special case of an A-pre-Rake combiner $X$ simplifies to

$$
X=\frac{1}{2 \pi} \sum_{m=1}^{M} \int_{-\pi}^{\pi}\left|\tilde{H}_{m}\left(e^{j \omega}\right)\right|^{2} \mathrm{~d} \omega,
$$

i.e., the effective SNR becomes

$$
\mathrm{SNR}=\frac{1}{2 \pi \sigma_{c}^{2}} \sum_{m=1}^{M} \int_{-\pi}^{\pi}\left|\tilde{H}_{m}\left(e^{j \omega}\right)\right|^{2} \mathrm{~d} \omega=\mathrm{SNR}_{\mathrm{MF}}
$$

Consequently, the proposed transmitter structure approaches the MF bound performance limit for sufficiently long spreading sequences. ${ }^{10}$ This result is intuitively pleasing since $N \rightarrow$ $\infty$ means that the overall CIR $q[N n]$ is ISI-free, and linear processing at the transmitter is optimum.

\section{UPLINK-DOWNLINK DUALITY}

In this section, we study a SIMO DS-UWB system with $M$ receive antennas, post-Rake combining, and postequalization, cf. Fig. 2. We will also refer to the SIMO system in Fig. 2 as the uplink to distinguish it from the MISO downlink configuration shown in Fig. 1. For $\tilde{h}_{m}[k]$ and $\tilde{g}_{m}[k]$ in Fig. 2 the definitions provided in Section II are still valid, i.e., $\tilde{h}_{m}[k]$ contains the combined effects of spreading with $c[N-1-k]$ and the CIR of antenna $m$, whereas $\tilde{g}_{m}[k]$ contains the combined effects of de-spreading with $c[k]$ and post-Rake combining. Each receive antenna employs a filter $f_{m}[n], 1 \leq m \leq M$, to remove residual ISI before a decision is made. The noise processes $z_{c, m}[k], 1 \leq m \leq M$, are mutually independent AWGN processes with variance $\sigma_{c}^{2}$.

\section{A. Filter Optimization}

In the following, we assume FIR filters $f_{m}[n], 1 \leq m \leq M$, and optimize them based on the MMSE criterion. We note that for $\boldsymbol{Q}, \boldsymbol{q}, \boldsymbol{\Phi}$, and $\boldsymbol{f}$ the same definitions as for the MISO downlink problem considered in Section III-A are valid. The receiver output signal $r[n]$ in Fig. 2 can be expressed as

$$
r[n]=(\boldsymbol{Q} \boldsymbol{f})^{H} \boldsymbol{a}[n]+\boldsymbol{f}^{H} \boldsymbol{z}[n],
$$

where

$$
\begin{gathered}
\boldsymbol{z}[n] \triangleq\left[\boldsymbol{z}_{1}^{T}[n] \ldots \boldsymbol{z}_{M}^{T}[n]\right]^{T} \\
\boldsymbol{z}_{m}[n] \triangleq\left[z_{m}[n] z_{m}[n-1] \ldots z_{m}\left[n-L_{f}+1\right]\right]^{T},
\end{gathered}
$$

${ }^{10}$ As will be seen in Section VI, depending on the channel model under consideration spreading sequences of length $N=25$ to $N=60$ already offer a performance that is virtually indistinguishable from the MF bound performance limit (cf. Fig. 5). 
and

$$
z_{m}[n]=\sum_{l=-\infty}^{\infty} z_{c, m}[l] \tilde{g}_{m}[N n-l] .
$$

Based on Eq. (32) the autocorrelation function of $z_{m}[n]$ can be calculated as

$$
\varphi_{z z}^{m}[n]=\mathcal{E}\left\{z_{m}[l+n] z_{m}^{*}[l]\right\}=\sigma_{c}^{2} \varphi_{m}[N n]
$$

with $\varphi_{m}[k]$ as defined in Section III-A. Exploiting this result we can express the error variance to be minimized as

$$
\begin{aligned}
\sigma_{e}^{2} & \triangleq \mathcal{E}\left\{\left|a\left[n-n_{0}\right]-r[n]\right|^{2}\right\} \\
& =1-\boldsymbol{f}^{H} \boldsymbol{q}-\boldsymbol{q}^{H} \boldsymbol{f}+\boldsymbol{f}^{H} \boldsymbol{Q}^{H} \boldsymbol{Q} \boldsymbol{f}+\sigma_{c}^{2} \boldsymbol{f}^{H} \boldsymbol{\Phi} \boldsymbol{f} .
\end{aligned}
$$

Minimizing $\sigma_{e}^{2}$ leads to the optimum vector

$$
\boldsymbol{f}_{\mathrm{opt}}^{\text {uplink }}=\left(\boldsymbol{Q}^{H} \boldsymbol{Q}+\sigma_{c}^{2} \boldsymbol{\Phi}\right)^{-1} \boldsymbol{q} .
$$

This means the optimum SIMO uplink post-equalization filters in Eq. (34) are up to an irrelevant scaling factor identical to the optimum MISO downlink PEFs. Furthermore, it can easily be verified that the minimum error variances are identical in both cases. In other words, there is a duality between SIMO MMSE post-equalization after post-Rake combining and MISO MMSE pre-equalization before pre-Rake combining. We note that this duality also holds if IIR MMSE equalization filters are employed, of course.

\section{B. Practical Implications}

The uplink-downlink duality of the considered MMSE problem is not only of theoretical interest but also has important practical implications. In particular, while adaptive algorithms cannot be used to calculate the optimum downlink filter $\boldsymbol{f}_{\text {opt }}$ since the receiver output signal $r[n]$ is not available at the transmitter, the uplink filter $f_{\text {opt }}^{\text {uplink }}$ can be efficiently calculated using an adaptive algorithm such as the least-mean square (LMS) or recursive least squares (RLS) algorithm [28]. For example, with the LMS algorithm the uplink filters are updated according to

$$
\boldsymbol{f}^{\text {uplink }}[n+1]=\boldsymbol{f}^{\text {uplink }}[n]+\mu_{0} e^{*}[n] \boldsymbol{y}[n],
$$

where $\mu_{0}$ and

$$
e[n] \triangleq a\left[n-n_{0}\right]-r[n]
$$

denote the adaptation step size and the error signal, respectively. Furthermore,

$$
\begin{gathered}
\boldsymbol{y}[n] \triangleq\left[\boldsymbol{y}_{1}^{T}[n] \ldots \boldsymbol{y}_{M}^{T}[n]\right]^{T}, \\
\boldsymbol{y}_{m}[n] \triangleq\left[y_{m}[n] y_{m}[n-1] \ldots y_{m}\left[n-L_{f}+1\right]\right]^{T},
\end{gathered}
$$

and $y_{m}[n]$ denotes the input signal for the equalization filter at antenna $m, 1 \leq m \leq M$, cf. Fig. 2. We note that for calculation of the error signal, $a\left[n-n_{0}\right]$ can either be a training symbol or a previously decided symbol. Once the LMS has converged and $\boldsymbol{f}_{\mathrm{opt}}^{\text {uplink }}$ is known, the normalization factor $\alpha$ required in the downlink can be obtained from

$$
\alpha_{\mathrm{opt}}=\sqrt{\left(\boldsymbol{f}_{\mathrm{opt}}^{\mathrm{uplink}}\right)^{H} \boldsymbol{\Phi} \boldsymbol{f}_{\mathrm{opt}}^{\mathrm{uplink}}},
$$

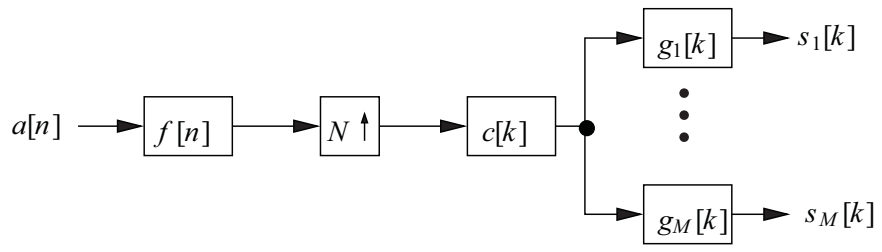

Fig. 3. Block diagram of MISO DS-UWB system (downlink) with $M$ transmit antennas, pre-Rake combining, and simplified pre-equalization.

and the optimum downlink PEF is ${ }^{11}$

$$
\boldsymbol{f}_{\mathrm{opt}}=\boldsymbol{f}_{\mathrm{opt}}^{\mathrm{uplink}} / \alpha_{\mathrm{opt}} \text {. }
$$

It should be noted that $\boldsymbol{\Phi}$ only depends on the pre-Rake filter coefficients. Therefore, the proposed recursive calculation of the optimum MISO downlink PEF $\boldsymbol{f}_{\text {opt }}$ only requires knowledge of the pre-Rake filter coefficients. For the P- and Spre-Rake estimation of the entire CIR $h_{m}[k], 0 \leq k \leq L_{h}$, $1 \leq m \leq M$, which is necessary for the closed-form solution in Eq. (12), can thus be avoided.

\section{Simplified PEF Structure}

In this section, we consider the simplified PEF (S-PEF) structure for the MISO downlink shown in Fig. 3. The main difference to the PEF structure shown in Fig. 1 is that only one PEF

$$
\boldsymbol{f} \triangleq\left[f[0] \ldots f\left[L_{f}-1\right]\right]^{T}
$$

is employed jointly for all $M>1$ transmit antennas, which reduces transmitter complexity.

\section{A. Filter Optimization}

As far as filter optimization is concerned, the S-PEF structure shown in Fig. 3 leads to an equivalent single-input single-output (SISO) channel with effective overall CIR

$$
q_{\mathrm{eff}}\left[N n+k_{0}\right]=\sum_{m=1}^{M} q_{m}\left[N n+k_{0}\right]
$$

and transmit power

$$
P=\boldsymbol{f}^{H} \boldsymbol{\Phi}_{\mathrm{eff}} \boldsymbol{f}
$$

where

$$
\boldsymbol{\Phi}_{\text {eff }}=\sum_{m=1}^{M} \boldsymbol{\Phi}_{m} .
$$

Consequently, based on the results in Section III-A the optimum FIR PEF is given by

$$
\begin{aligned}
& \boldsymbol{f}_{\mathrm{opt}}=\frac{1}{\alpha_{\mathrm{opt}}^{*}} \boldsymbol{V} \boldsymbol{q}_{\mathrm{eff}} \\
& \alpha_{\mathrm{opt}}=\sqrt{\boldsymbol{q}_{\mathrm{eff}}^{H} \boldsymbol{V} \boldsymbol{\Phi}_{\mathrm{eff}} \boldsymbol{V} \boldsymbol{q}_{\mathrm{eff}}},
\end{aligned}
$$

where

$$
\begin{gathered}
\boldsymbol{V} \triangleq\left(\boldsymbol{Q}_{\mathrm{eff}}^{H} \boldsymbol{Q}_{\mathrm{eff}}+\sigma_{c}^{2} \boldsymbol{\Phi}_{\mathrm{eff}}\right)^{-1}, \\
\boldsymbol{q}_{\mathrm{eff}} \triangleq \boldsymbol{Q}_{\mathrm{eff}}^{H} \boldsymbol{e}_{n_{0}},
\end{gathered}
$$

\footnotetext{
${ }^{11}$ Note that the normalization with $\alpha_{\mathrm{opt}}$ is necessary to meet the transmit power constraint, cf. Eq. (7).
} 
and

$$
\boldsymbol{Q}_{\mathrm{eff}} \triangleq \sum_{m=1}^{M} \boldsymbol{Q}_{m}
$$

The corresponding minimum error variance is obtained by replacing $\boldsymbol{q}, \boldsymbol{Q}$, and $\Phi$ in Eq. (14) by $\boldsymbol{q}_{\mathrm{eff}}, \boldsymbol{Q}_{\mathrm{eff}}$, and $\boldsymbol{\Phi}_{\mathrm{eff}}$, respectively. Similarly, in the IIR case we obtain

$$
\begin{aligned}
F_{\mathrm{opt}}\left(e^{j \omega}\right) & =\frac{1}{\alpha_{\mathrm{opt}}^{*}} \frac{Q_{\mathrm{eff}}\left(e^{j \omega}\right)}{\Phi_{\mathrm{eff}}\left(e^{j \omega}\right)\left(\sigma_{c}^{2}+X_{\mathrm{eff}}\left(e^{j \omega}\right)\right)} \\
\alpha_{\mathrm{opt}} & =\sqrt{\frac{1}{2 \pi} \int_{-\pi}^{\pi} \frac{X_{\mathrm{eff}}\left(e^{j \omega}\right)}{\left(\sigma_{c}^{2}+X_{\mathrm{eff}}\left(e^{j \omega}\right)\right)^{2}} \mathrm{~d} \omega},
\end{aligned}
$$

where

$$
\begin{gathered}
X_{\mathrm{eff}}\left(e^{j \omega}\right)=\frac{\left|Q_{\mathrm{eff}}\left(e^{j \omega}\right)\right|^{2}}{\Phi_{\mathrm{eff}}\left(e^{j \omega}\right)}, \\
Q_{\mathrm{eff}}\left(e^{j \omega}\right) \triangleq \sum_{m=1}^{M} Q_{m}\left(e^{j \omega}\right),
\end{gathered}
$$

and

$$
\Phi_{\mathrm{eff}}\left(e^{j \omega}\right) \triangleq \sum_{m=1}^{M} \Phi_{m}\left(e^{j \omega}\right),
$$

cf. Section III-B. The corresponding error variance is given by

$$
\sigma_{e, \min }^{2}=\frac{1}{2 \pi} \int_{-\pi}^{\pi} \frac{\sigma_{c}^{2}}{\sigma_{c}^{2}+X_{\mathrm{eff}}\left(e^{j \omega}\right)} \mathrm{d} \omega .
$$

The approximate BER of the S-PEF scheme can be obtained by applying $\sigma_{e, \min }^{2}$ from Eq. (40) (or the corresponding expression for the FIR case) in Eq. (24). Furthermore, we note that an uplink-downlink duality can also be established for the simplified MISO downlink structure and a corresponding simplified SIMO uplink configuration with just one postequalization filter. Using similar steps as in Section IV-B for the original transmitter structure, this duality can be exploited for adaptive calculation of the optimum PEF.

\section{B. Comparison}

It is of interest to compare the performances of the transmitter structures in Figs. 1 and 3 for IIR PEFs. For this purpose, we first note that based on the complex version of Hölder's inequality [29] we can establish the following inequality

$$
\begin{array}{r}
\left(\sum_{m=1}^{M}\left|\sqrt{\Phi_{m}\left(e^{j \omega}\right)}\right|^{2}\right)^{1 / 2}\left(\sum_{m=1}^{M}\left|\frac{Q_{m}\left(e^{j \omega}\right)}{\sqrt{\Phi_{m}\left(e^{j \omega}\right)}}\right|^{2}\right)^{1 / 2} \geq \\
\left|\sum_{m=1}^{M} Q_{m}\left(e^{j \omega}\right)\right| .
\end{array}
$$

Squaring both sides of Eq. (41) and dividing them subsequently by $\sum_{m=1}^{M} \Phi_{m}\left(e^{j \omega}\right)$ leads to

$$
\begin{gathered}
X_{\mathrm{eff}}\left(e^{j \omega}\right)=\frac{\left|\sum_{m=1}^{M} Q_{m}\left(e^{j \omega}\right)\right|^{2}}{\sum_{m=1}^{M} \Phi_{m}\left(e^{j \omega}\right)} \leq \\
\sum_{m=1}^{M} \frac{\left|Q_{m}\left(e^{j \omega}\right)\right|^{2}}{\Phi_{m}\left(e^{j \omega}\right)}=X\left(e^{j \omega}\right),
\end{gathered}
$$

where $X\left(e^{j \omega}\right)$ is defined in Eq. (25). Since $X\left(e^{j \omega}\right)$ and $X_{\text {eff }}\left(e^{j \omega}\right)$ appear in the denominator of the respective error variances, cf. Eqs. (22), (40), Eq. (42) shows that the S-PEF scheme cannot outperform the PEF scheme. This result is not surprising since the S-PEF structure in Fig. 3 may be viewed as a special case of the PEF structure in Fig. 1 with

$$
f_{1}[n]=f_{2}[n]=\cdots=f_{M}[n], \quad 0 \leq n<L_{f} .
$$

For the special case of an A-pre-Rake filter Eq. (42) simplifies to

$$
X_{\text {eff }}\left(e^{j \omega}\right)=\frac{1}{N} \sum_{m=1}^{M} \sum_{k=0}^{N-1}\left|\tilde{H}_{m}\left(e^{j(\omega-2 \pi k) / N}\right)\right|^{2}=X\left(e^{j \omega}\right) .
$$

It follows from Eq. (43) that the minimum error variances for the S-PEF and the PEF scheme are equal in this case, cf. Eqs. (22), (40), i.e., both schemes will achieve the same performance. Therefore, the S-PEF and the PEF schemes are equivalent for IIR PEFs and A-pre-Rake combining, which implies that the S-PEF scheme should perform close to optimum as long as sufficiently long FIR PEFs and a good approximation of the A-pre-Rake filter (i.e., a P- or S-preRake filter with a sufficient number of fingers) are employed. ${ }^{12}$ Thus, in this case, the more complex structure in Fig. 1 can be avoided. On the other hand, if a suboptimum pre-Rake filter with very few fingers and/or short FIR PEFs are used, the PEF structure in Fig. 1 is preferable and will lead to a better performance than the S-PEF structure in Fig. 3.

\section{Simulation and Numerical Results}

In this section, we present computer simulation and numerical results for the proposed PEF schemes for MISO DS-UWB systems. Thereby, we consider the practically most relevant cases of $M=1$ and $M=2$ transmit antennas and adopt the channel model discussed in Section II-B. In particular, we focus on CM1 and CM4 since they have the smallest and the largest average delay spread of the four channel models, respectively. For convenience and practical relevance, we adopted for our simulations the parameters from the IEEE 802.15.3a standardization efforts. In particular, we assume a chip duration of $T_{c}=0.76 \mathrm{~ns}$ and both transmit filter $g_{T}(t)$ and receive filter $g_{R}(t)$ are square-root raised-cosine filters with roll-off factor 0.3 [30]. For $M=2$ transmit antennas we assumed that the lognormal terms $X_{m}, m \in\{1,2\}$, are correlated with correlation coefficient $\rho=0.86$ [21]. All effective SNR and BER results shown in the following were averaged over 100 channel realizations. For our simulations, we generated oversampled versions of $h_{m}^{\prime}(t), h_{m}(t), g_{T}(t)$, and $g_{R}(t)$ with a sampling interval of $T_{c} / 8$, respectively. The oversampled overall CIR was then down-sampled and the resulting overall discrete-time CIR was truncated to a length $L_{h}$ such that

$$
\frac{\sum_{k=0}^{L_{h}-1} \mathcal{E}\left\{\left|h_{m}[k]\right|^{2}\right\}}{\sum_{k=-\infty}^{\infty} \mathcal{E}\left\{\left|h_{m}[k]\right|^{2}\right\}}>0.999 .
$$

\footnotetext{
${ }^{12}$ As will be seen in Section VI for the example of channel model CM4, when employing PEFs of length $L_{f}=100$ in conjunction with an A-preRake filter, the performances of the PEF and the S-PEF schemes are virtually the same. However, an S-pre-Rake filter with $S=32$ fingers already leads to a small performance gap (cf. Fig. 6). The same effect can be observed if PEFs of smaller lengths are employed.
} 


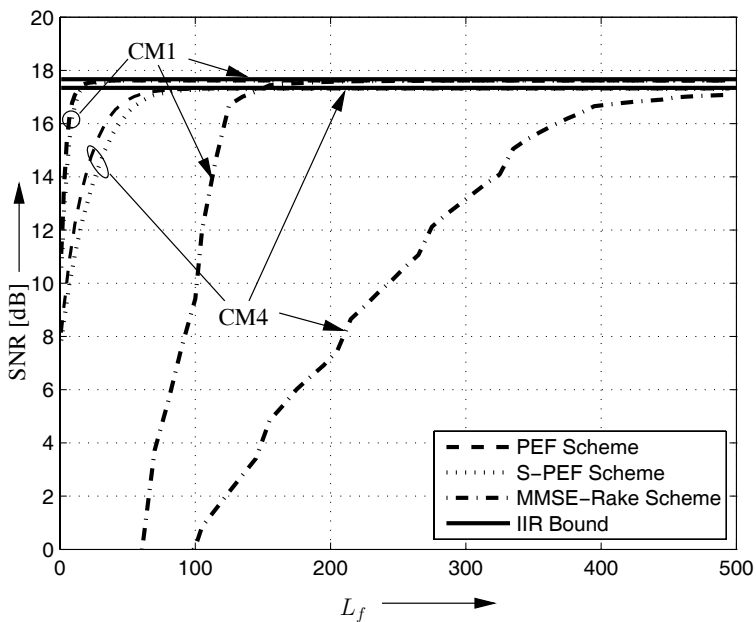

Fig. 4. Effective SNR vs. $L_{f}$ for PEF, S-PEF, and MMSE-Rake [15] schemes for CM1 and CM4 UWB channel models. All results are averaged over 100 channel realizations. A-pre-Rake, $M=2, N=6$, and $E_{b} / N_{0}=$ $15 \mathrm{~dB}$.

This led to $L_{h}=140$ and $L_{h}=420$ for CM1 and CM4, respectively. Throughout this section we adopt for the sampling phase $k_{0}=k_{0}^{s}$, cf. Section II-C, and for FIR PEFs the decision delay $n_{0}$ was optimized.

\section{A. Effective SNR Results}

First, we show numerical results for the effective SNR at the receiver $\left(\mathrm{SNR}=1 / \sigma_{e, \min }^{2}-1\right)$. Here, $\sigma_{e, \min }^{2}$ is obtained from the analytical expressions in Eqs. (14), (22), and (40).

Fig. 4 shows the effective SNR of the PEF and S-PEF schemes with A-pre-Rake combining vs. FIR PEF length $L_{f}$ for CM1 and CM4, where $M=2, N=6$, and $E_{b} / N_{0} \triangleq$ $1 / \sigma_{c}^{2}=15 \mathrm{~dB}$. Here, $E_{b}$ and $N_{0}$ denote the average energy per bit and the single-sided power spectral density of the underlying passband AWGN process, respectively. Fig. 4 shows that as $L_{f}$ increases the FIR PEF filters quickly approach the performance of the IIR PEF filters (solid lines). Since the average delay spread for CM1 (and thus the resulting CIR length $L_{h}$ ) is considerably smaller than for CM4, this convergence is much faster for CM1 than that for CM4. We also note that while the PEF scheme achieves a higher SNR than the S-PEF scheme for short FIR PEFs, both schemes achieve the same performance for long FIR and IIR filters, cf. Section V-B. For comparison, we have also included in Fig. 4 the results for the MMSE-Rake scheme proposed in [15]. As $L_{f}$ increases the MMSE-Rake scheme achieves the same performance as the proposed PEF and S-PEF schemes. However, since the filters in the MMSE-Rake scheme operate at the chip level, the convergence to the optimum IIR performance is much slower than for the PEF and S-PEF schemes. For example, if an SNR of $14 \mathrm{~dB}$ is desired for CM4, the PEF and the MMSE-Rake schemes require filter lengths of 18 and 325, respectively. The computation of the long filters required for the MMSE-Rake scheme may be very difficult in practice even if a recursive (e.g. steepest descent) or adaptive (e.g. LMS) algorithm is used to avoid direct matrix inversion.

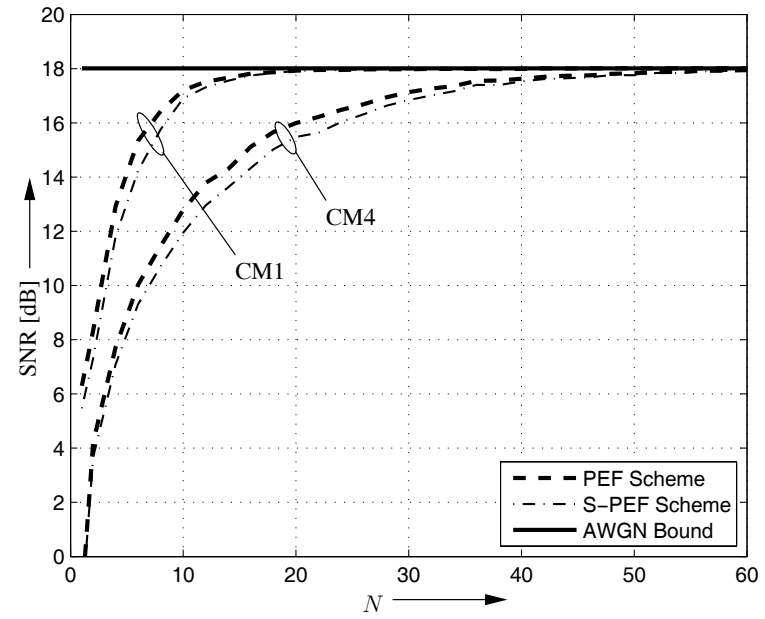

Fig. 5. Effective SNR vs. $N$ for PEF and S-PEF schemes for CM1 and CM4 UWB channel models. All results are averaged over 100 channel realizations. A-pre-Rake, $M=2, L_{f}=5$, and $E_{b} / N_{0}=15 \mathrm{~dB}$.

In Fig. 5, we investigate the dependence of the effective SNR of the PEF and S-PEF schemes on the spreading factor $N$ for CM1 and CM4. $M=2, L_{f}=5, E_{b} / N_{0}=15 \mathrm{~dB}$, and A-pre-Rake combining are assumed. As $N$ increases the effective amount of ISI after pre-Rake combining rapidly decreases, and both the PEF and S-PEF schemes approach the $\mathrm{SNR}$ of the pure AWGN channel given by $\mathrm{SNR}=M / \sigma_{c}^{2}$ (solid line), cf. Section III-F. Again the convergence to the optimum value is slower for CM4 than for CM1 because of its larger delay spread, and the PEF scheme outperforms the S-PEF scheme for small values of $N$.

The effective SNR of the PEF and S-PEF schemes as a function of $L_{f}$ is shown in Fig. 6 for A-pre-Rake combining and S-pre-Rake combining with different numbers of fingers $S$. CM4, $M=2, N=6$, and $E_{b} / N_{0}=15 \mathrm{~dB}$ are employed. As predicted in Section V-B, with S-pre-Rake combining the PEF scheme outperforms the S-PEF scheme even for IIR PEFs and the performance gap between both schemes increases as the number of fingers decreases. For example, the asymptotic SNR differences between the PEF and S-PEF schemes for $S=32,16$, and 8 are $0.25 \mathrm{~dB}, 0.62 \mathrm{~dB}$, and 1.2 $\mathrm{dB}$, respectively.

\section{B. BER Results}

Next, we show simulation and numerical results for the BER performance of the PEF and S-PEF schemes. CM4 is assumed for all results shown in this section.

Fig. 7 shows simulated BER results for the PEF and S-PEF schemes with FIR PEFs of lengths $L_{f}=5,10$, and 20 and numerical results for the same schemes with IIR PEFs obtained from Eq. (24). $M=2, N=6$, and Apre-Rake combining are assumed. For comparison we also show simulation results for the A-pre-Rake (or time-reversal) scheme without pre-equalization as well as the MF bound calculated based on Eq. (28). As can be observed from Fig. 7, both the PEF and S-PEF schemes significantly lower the high BER floor of the pure A-pre-Rake scheme. The performance 


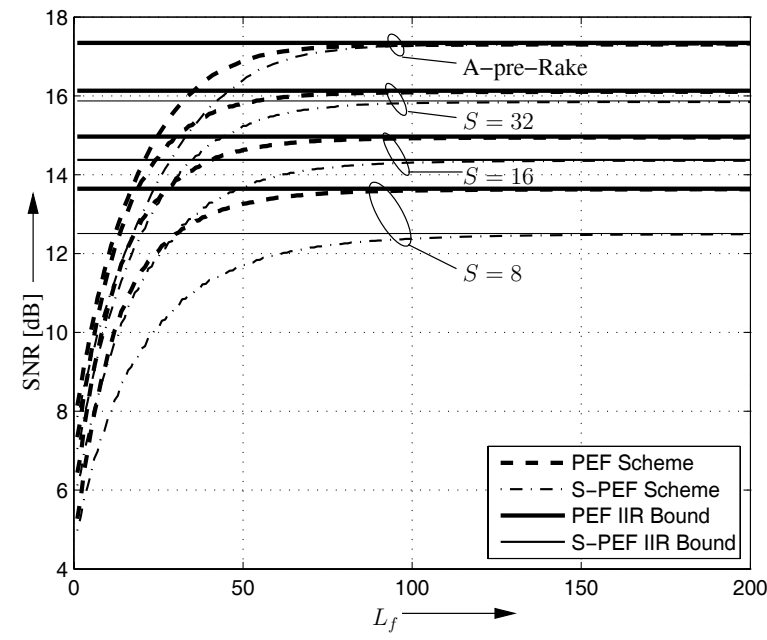

Fig. 6. Effective SNR vs. $L_{f}$ for PEF and S-PEF schemes for CM4 UWB channel model. All results are averaged over 100 channel realizations. S-preRake, $M=2, N=6$, and $E_{b} / N_{0}=15 \mathrm{~dB}$.

gap between the PEF scheme and the S-PEF scheme decreases as $L_{f}$ increases and disappears for $L_{f} \rightarrow \infty$, as expected from the discussion in Section V-B. We note that even for IIR PEFs there remains a $1-\mathrm{dB}$ gap to the MF bound. However, to further narrow this gap some form of non-linear processing at the transmitter would be required, which would (further) increase complexity.

In Fig. 8, we compare the performances of the PEF scheme for $M=1$ and $M=2$ transmit antennas assuming S-preRake combining $(S=16)$ and $N=6$. The BER curves for the FIR PEFs and the S-pre-Rake scheme without equalization were simulated, whereas the BER curves for the IIR PEFs and the MF bound were obtained by evaluating Eqs. (24) and (28), respectively. Fig. 8 shows that a second transmit antenna yields substantial performance improvements even if the corresponding lognormal shadowing terms are correlated. This performance gain is about $2.6 \mathrm{~dB}$ for IIR PEFs (at a BER of $10^{-6}$ ), and even larger gains are obtained for short FIR PEFs. These gains are due to the fact that increasing $M$ has a similar effect as increasing the spreading factor $N$, cf. Section III-D. Remarkably, even if we fix the total number of FIR filter taps $M L_{f}$, the SISO scheme with $L_{f}=10$ and $L_{f}=20$ performs substantially worse than the MISO scheme with $L_{f}=5$ and $L_{f}=10$, respectively. The relatively large gap between the MF bounds and the corresponding PEF scheme with IIR filters is due to the suboptimum S-pre-Rake combining.

In Fig. 9, we compare the performance of the PEF and S-PEF schemes for different spreading factors $N$ assuming S-pre-Rake combining $(S=16)$ and $M=2$. For FIR PEFs with $L_{f}=10$ both simulation (markers) and analytical (lines) results obtained from Eq. (24) are shown. For IIR PEFs only analytical results for $N=12$ are shown. For the FIR PEFs the analytical and simulation results are in good agreement. Furthermore, since the amount of ISI after pre-Rake combining decreases as $N$ increases, the performance of both the PEF and S-PEF schemes improves and the gap between

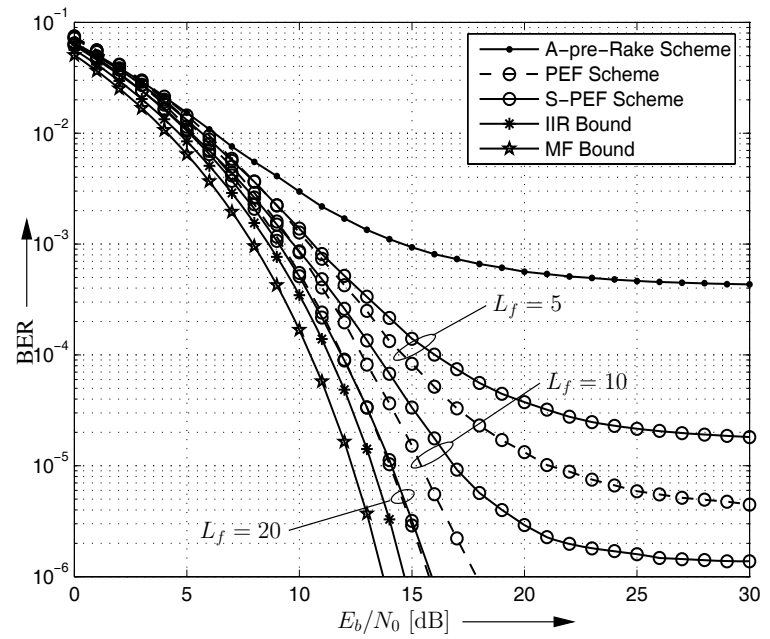

Fig. 7. BER vs. $E_{b} / N_{0}$ for PEF, S-PEF, and A-pre-Rake schemes for CM4. All results are averaged over 100 channel realizations. A-pre-Rake, $M=2$, and $N=6$. MF bound is also shown.

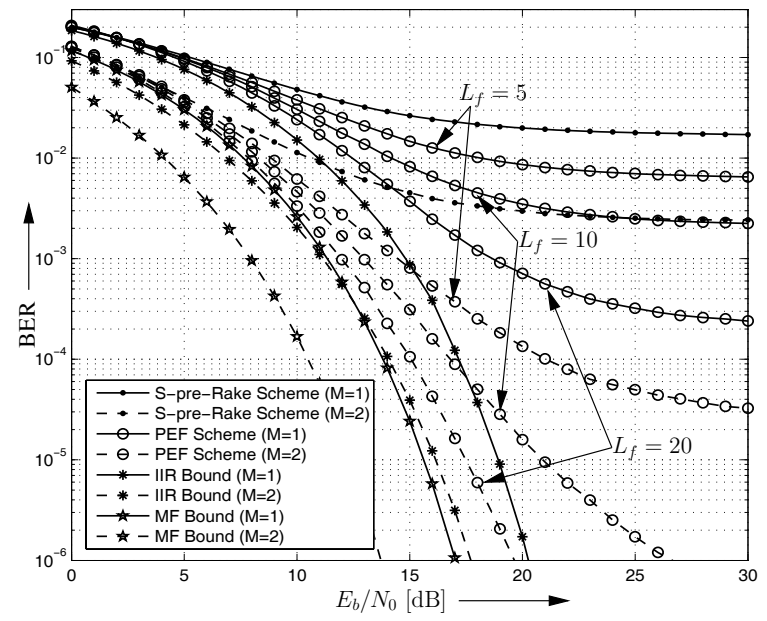

Fig. 8. BER vs. $E_{b} / N_{0}$ for PEF and S-pre-Rake schemes for CM4. All results are averaged over 100 channel realizations. S-pre-Rake with $S=16$ fingers and $N=6$. MF bounds are also shown.

both schemes decreases with increasing $N$. Of course, this performance improvement comes at the price of a decreased data rate. We note that since S-pre-Rake combining is used the PEF scheme outperforms the S-PEF scheme even for IIR PEFs, cf. Section V-B. Furthermore, if the number of fingers $S$ was reduced, the gap between both schemes would further increase, cf. Fig. 6.

\section{CONCLUSIONS}

In this paper, we have proposed two different PEF schemes for MISO DS-UWB systems with pre-Rake combining. The first PEF scheme employs one PEF per transmit antenna, whereas the second scheme, the simplified PEF scheme, requires only one PEF. In contrast to previously proposed prefiltering schemes for DS-UWB, both proposed PEF schemes efficiently exploit the channel shortening properties of the pre-Rake filter and operate at the symbol level. Therefore, 


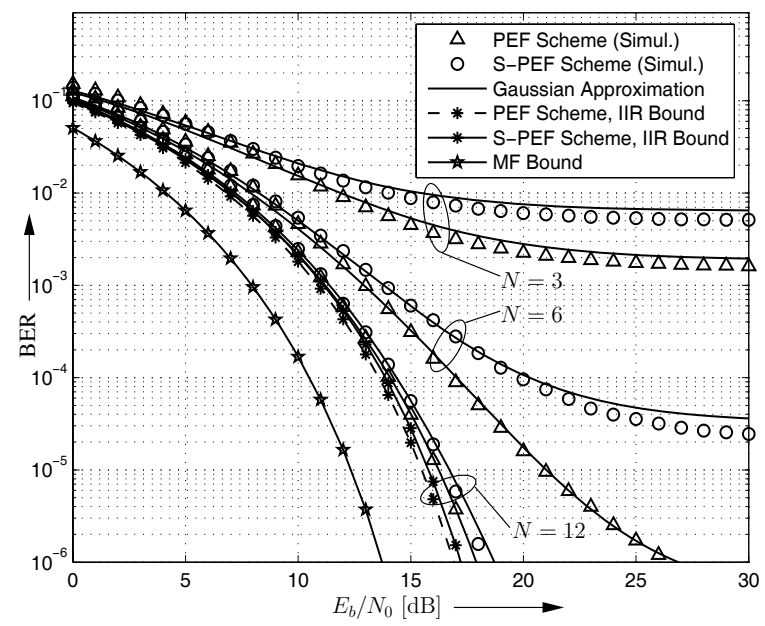

Fig. 9. BER vs. $E_{b} / N_{0}$ for PEF and S-PEF schemes for CM4. All results are averaged over 100 channel realizations. S-pre-Rake with $S=16$ fingers, $M=2$, and $L_{f}=10$. MF bound is also shown.

relatively short PEFs achieve close-to-optimum performance even for long UWB CIRs. For sufficiently long PEFs and A-pre-Rake combining both proposed PEF schemes achieve the same performance. However, the S-PEF scheme suffers from a certain performance degradation for suboptimum preRake combining and/or short PEFs. Furthermore, we have also shown that a SIMO DS-UWB system with post-Rake combining and MMSE post-equalization is the dual system for a MISO DS-UWB system with pre-Rake combining and MMSE pre-equalization. This uplink-downlink duality can be exploited for complexity reduction. Simulation results have confirmed the analytical findings and the excellent performance of the proposed PEF schemes.

We note that while in this paper only DS-UWB systems have been considered, the proposed PEF schemes are also applicable to other areas (e.g. TDD-CDMA systems and underwater acoustic communication) where pre-Rake combining is used.

\section{Appendix A: Average Transmit Power}

In this appendix, we evaluate Eq. (7) for FIR and IIR PEFs, respectively.

FIR Case: Based on Eq. (2) we obtain

$\mathcal{E}\left\{\left|s_{m}[k]\right|^{2}\right\}=\sum_{i=-\infty}^{\infty} \sum_{j=-\infty}^{\infty} \varphi_{f f}^{m}[i-j] \tilde{g}_{m}[k-i N] \tilde{g}_{m}^{*}[k-j N]$,

where

$$
\varphi_{f f}^{m}[k] \triangleq f_{m}[k] * f_{m}^{*}[-k] .
$$

Eq. (44) shows that $s_{m}[k]$ is a cyclo-stationary process with period $N$. The signal power

$$
P_{m} \triangleq \sum_{k=N n}^{N(n+1)-1} \mathcal{E}\left\{\left|s_{m}[k]\right|^{2}\right\}
$$

per symbol interval at antenna $m$ can be obtained as

$$
\begin{aligned}
P_{m}= & \sum_{i=-\infty}^{\infty} \sum_{j=-\infty}^{\infty} \sum_{k=N n}^{N(n+1)-1} \varphi_{f f}^{m}[i-j] \tilde{g}_{m}[k-i N] \tilde{g}_{m}^{*}[k-j N] \\
= & \sum_{l=-\infty}^{\infty} \varphi_{f f}^{m}[l] \sum_{j=-\infty}^{\infty} \sum_{k=N n}^{N(n+1)-1} \tilde{g}_{m}[k-j N-l N] \tilde{g}_{m}^{*}[k-j N] \\
= & \sum_{l=-\infty}^{\infty} \varphi_{f f}^{m}[l] \varphi_{m}[-N l],
\end{aligned}
$$

where

$$
\varphi_{m}[k] \triangleq \tilde{g}_{m}[k] * \tilde{g}_{m}^{*}[-k]
$$

is defined in Section III-A. Now, $P_{m}$ can be rewritten in vector form as

$$
P_{m}=\boldsymbol{f}_{m}^{H} \boldsymbol{\Phi}_{m} \boldsymbol{f}_{m}
$$

where $\boldsymbol{\Phi}_{m}$ is also defined in Section III-A. The total average transmit power per symbol interval is then

$$
P=\sum_{m=1}^{M} P_{m}
$$

which results in Eq. (10).

IIR Case: Based on Eq. (45) we may rewrite the signal power for antenna $m$ as

$$
\begin{aligned}
P_{m} & =\frac{1}{2 \pi} \sum_{l=-\infty}^{\infty} \int_{-\pi}^{\pi} \Phi_{f f}^{m}\left(e^{j \omega}\right) \varphi_{m}[-N l] e^{j \omega l} \mathrm{~d} \omega \\
& =\frac{1}{2 \pi} \int_{-\pi}^{\pi}\left|F_{m}\left(e^{j \omega}\right)\right|^{2} \Phi_{m}\left(e^{j \omega}\right) \mathrm{d} \omega,
\end{aligned}
$$

where we have used the definition of the (inverse) Fourier transform and the identity

$$
\Phi_{f f}^{m}\left(e^{j \omega}\right) \triangleq \mathcal{F}\left\{\varphi_{f f}^{m}[k]\right\}=\left|F_{m}\left(e^{j \omega}\right)\right|^{2} .
$$

Eq. (17) is now easily obtained by adding the powers $P_{m}$, $1 \leq m \leq M$, of all $M$ antennas.

\section{APPENDIX B: ERROR VARIANCE FOR IIR PEFS}

For IIR PEFs without causality constraint the definitions of $\boldsymbol{f}_{m}, \boldsymbol{q}_{m}$, and $\boldsymbol{Q}_{m}$ have to be slightly modified compared to the FIR case with causality constraint. For example, now

$$
\boldsymbol{f}_{m} \triangleq\left[\ldots f_{m}[-1] f_{m}[0] f_{m}[1] \ldots\right]^{T}
$$

and

$$
\boldsymbol{q}_{m} \triangleq\left[\ldots q_{m}\left[-N+k_{0}\right] q_{m}\left[k_{0}\right] q_{m}\left[N+k_{0}\right] \ldots\right]^{T}
$$


are valid. Taking these changes into account, $\boldsymbol{f}^{H} \boldsymbol{q}$ can be expressed as

$$
\begin{aligned}
\boldsymbol{f}^{H} \boldsymbol{q} & =\sum_{m=1}^{M} \sum_{n=-\infty}^{\infty} f_{m}[n] q_{m}\left[-N n+k_{0}\right] \\
& =\frac{1}{2 \pi} \sum_{m=1}^{M} \sum_{n=-\infty}^{\infty} \int_{-\pi}^{\pi} F_{m}\left(e^{j \omega}\right) q_{m}\left[-N n+k_{0}\right] e^{j \omega n} \mathrm{~d} \omega \\
& =\frac{1}{2 \pi} \int_{-\pi}^{\pi} \sum_{m=1}^{M} F_{m}\left(e^{j \omega}\right) Q_{m}\left(e^{j \omega}\right) \mathrm{d} \omega \\
& =\frac{1}{2 \pi} \int_{-\pi}^{\pi} \boldsymbol{F}^{H}\left(e^{j \omega}\right) \boldsymbol{Q}\left(e^{j \omega}\right) \mathrm{d} \omega,
\end{aligned}
$$

where we have used the definition of the (inverse) discretetime Fourier transform. Taking the non-causality and IIR property into account in the definition of $\boldsymbol{Q}_{m}, \boldsymbol{f}^{H} \boldsymbol{Q}^{H} \boldsymbol{Q} \boldsymbol{f}$ can be rewritten as

$$
\begin{aligned}
\boldsymbol{f}^{H} \boldsymbol{Q}^{H} \boldsymbol{Q} \boldsymbol{f} & =\sum_{m=1}^{M}\left\|\boldsymbol{Q}_{m} \boldsymbol{f}_{m}\right\|^{2} \\
& =\sum_{m=1}^{M} \sum_{n=-\infty}^{\infty}\left|x_{m}[n]\right|^{2} \\
& =\frac{1}{2 \pi} \int_{-\pi}^{\pi} \sum_{m=1}^{M}\left|X_{m}\left(e^{j \omega}\right)\right|^{2} \mathrm{~d} \omega \\
& =\frac{1}{2 \pi} \int_{-\pi}^{\pi} \boldsymbol{F}^{H}\left(e^{j \omega}\right) \boldsymbol{Q}\left(e^{j \omega}\right) \boldsymbol{Q}^{H}\left(e^{j \omega}\right) \boldsymbol{F}\left(e^{j \omega}\right) \mathrm{d} \omega,
\end{aligned}
$$

where we have employed the definitions

$$
\begin{gathered}
x_{m}[n] \triangleq f_{m}[n] * q_{m}\left[N n+k_{0}\right], \\
X_{m}\left(e^{j \omega}\right) \triangleq \mathcal{F}\left\{x_{m}[n]\right\}=F_{m}\left(e^{j \omega}\right) Q_{m}\left(e^{j \omega}\right),
\end{gathered}
$$

and Parseval's theorem [24]. Applying Eqs. (47) and (48) in Eq. (9), Eq. (16) directly follows.

\section{REFERENCES}

[1] M. Win and R. Scholtz, "Impulse radio: how it works," IEEE Commun. Lett., vol. 2, pp. 36-38, Feb. 1998.

[2] M. Win and R. Scholtz, "Characterization of ultra-wide bandwidth wireless indoor channels: a communication-theoretic view," IEEE $J$. Select. Areas Commun., vol. 20, pp. 1613-1627, Dec. 2002.

[3] R. Esmailzadeh, E. Sourour, and M. Nakagawa, "PreRAKE diversity combining in time-division duplex CDMA mobile communications," IEEE Trans. Veh. Technol., vol. 48, pp. 795-801, May 1999.

[4] A. Parvulescu, "Matched-signal ('MESS') processing by the ocean," J. Acoust. Soc. Am., vol. 98, pp. 943-960, Aug. 1995.

[5] T. Strohmer, M. Emami, J. Hansen, G. Papanicolaou, and A. Paulraj, "Application of time-reversal with MMSE equalizer to UWB communications," in Proc. IEEE Global Telecomm. Conf. (Globecom), pp. 3123 3127, Nov. 2004

[6] S. Imada and T. Ohtsuki, "Pre-Rake diversity combining for UWB systems in IEEE 802.15 UWB multipath channel," in Proc. Joint Ultra Wideband Systems and Technology and Intern. Workshop for Ultra Wideband Systems, pp. 236-240, May 2004.

[7] K. Usuda, H. Zhang, and M. Nakagawa, "Pre-Rake performance for pulse based UWB system in a standardized UWB short-range channel," in Proc. IEEE Wireless Commun. and Networking Conf. (WCNC), pp. 920-925, Mar. 2004.
[8] N. Guo, R. Qiu, and B. Sadler, "An ultra-wideband autocorrelation demodulation scheme with low-complexity time reversal enhancement," in Proc. IEEE Military Commun. Conf. (Milcom), pp. 3066-3072, Atlantic City, Oct. 2005.

[9] H. Nguyen, I. Kovacs, and P. Eggers, "A time reversal transmission approach for multiuser UWB communications," IEEE Trans. Antennas Propag., vol. 54, pp. 3216-3224, Nov. 2006.

[10] Y. Nishida, C. Fukao, M. Fujii, M. Itami, and K. Itoh, "A study on improving performance of pre-post-RAKE combining in UWB-IR system," in Proc. IEEE Intern. Conf. Ultra-Wideband (ICUWB), pp. 79-84, Sept. 2006.

[11] R. Qiu, C. Zhou, N. Guo, and J. Zhang, "Time reversal with MISO for ultrawideband communications: experimental results," IEEE Antennas and Wireless Propag. Lett., vol. 5, pp. 269-273, Dec. 2006.

[12] W. Cao, A. Nallanathan, and C. C. Chai, "Performance analysis of prerake DS UWB multiple access system under imperfect channel estimation," IEEE Trans. Wireless Commun., vol. 6, pp. 3892-3896, Nov. 2007.

[13] Y.-H. Chang, S.-H. Tsai, X. Yu, and C.-C. Kuo, "Ultrawideband transceiver design using channel phase precoding," IEEE Trans. Signal Processing, vol. 55, pp. 3807-3822, July 2007.

[14] G. Ding, D. Wang, and Z. Chen, "Performance evaluation of two kinds of precoding joint with MMSE equalization in DS-UWB," in Proc. First Intern. Conf. Commun. and Networking in China (ChinaCom), Beijing, Oct. 2006.

[15] M. Emami, M. Vu, J. Hansen, A. Paulraj, and G. Papanicolaou, "Matched filtering with rate back-off for low complexity communications in very large delay spread channels," in Proc. 38th Asilomar Conf. Signals, Systems, and Computers, pp. 218-222, Nov. 2004.

[16] T. Berger and D. W. Tufts, "Optimum pulse amplitude modulationpart I: transmitter-receiver design and bounds from information theory," IEEE Trans. Inform. Theory, vol. IT-13, pp. 196-208, Apr. 1967.

[17] J. Yang and S. Roy, "On joint transmitter and receiver optimization for multi-input multi-output (MIMO) transmission systems," IEEE Trans. Commun., vol. COM-42, pp. 3221-3231, Dec. 1994.

[18] D. Tse and P. Viswanath, Fundamentals of Wireless Communication. Cambridge Univeristy Press, 2005.

[19] Channel modeling sub-committee final report, IEEE 802.15-02/368r5SG3a, IEEE P802.15, Dec. 2002.

[20] A. Molisch, J. Foerster, and M. Pendergrass, "Channel models for ultrawideband personal area networks," IEEE Wireless Commun., vol. 10, pp. 14-21, Dec. 2003.

[21] Z. Lin, X. Peng, K. Png, and F. Chin, "Kronecker modelling for correlated shadowing in UWB MIMO channels," in Proc. IEEE Wireless Commun. and Networking Conf. (WCNC), Hong Kong, Mar. 2007.

[22] P. A. Voois, I. Lee, and J. M. Cioffi, "The effect of decision delay in finite-length decision feedback equalization," IEEE Trans. Inform. Theory, vol. IT-42, pp. 618-621, Mar. 1996.

[23] J. G. Proakis, Digital Communications, 4th edition. New York: McGraw-Hill, 2001.

[24] A. V. Oppenheim and A. S. Willsky, Signals and Systems. Upper Saddle River, NJ: Prentice-Hall, Inc., 1996.

[25] T. K. Moon and W. C. Stirling, Mathematical Methods and Algorithms for Signal Processing. New York: Prentice Hall, 2000.

[26] V. Poor and S. Verdu, "Probability of error in MMSE multiuser detection," IEEE Trans. Inform. Theory, vol. 43, pp. 858-871, May 1997.

[27] B. Hu and N. Beaulieu, "Comparison of modulation schemes and Rake receiver structures for UWB systems on an IEEE 802.15.3 indoor channel," in Proc. IEEE Global Telecommun. Conf. (Globecom), pp. 3493-3497, Nov. 2005.

[28] S. Haykin, Adaptive Filter Theory, 3rd ed. Upper Saddle River, NJ: Prentice-Hall, 1996.

[29] I. S. Gradshteyn and I. M. Ryzhik, Table of Integrals, Series, and Products. New York: Academic Press, 2000.

[30] R. Fisher, R. Kohno, M. McLaughlin, and M. Welbourn, DS-UWB physical layer submission to IEEE 802.15 task group 3a (doc. number P802.15-03/0137r4), Jan. 2005. 


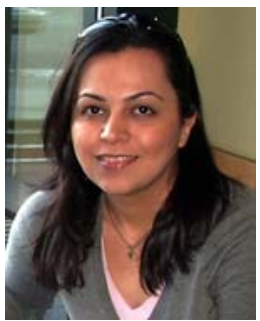

Elham Torabi (S'07) received her B.A.Sc. degree in electrical engineering from the University of Tehran, Tehran, Iran in 1997 and her M.A.Sc. degree in electrical engineering from the University of British Columbia, Vancouver, Canada in 2007. From 1997 to 2003 she was with Informatics Services Corporation, Tehran, Iran, where she worked as a communication system engineer for Hughes integrated satellite networks. Her current research interests include wireless communication systems and networks and ultra wideband technology.

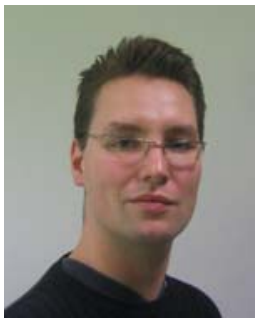

Jan Mietzner (S'02-M'08) was born in Rendsburg, Germany, on March 6, 1975. He studied electrical engineering and information engineering at the Faculty of Engineering, Christian-Albrechts University (CAU) of Kiel, Germany, with focus on digital communications. During his studies, he spent six months in 2000 with the Global Wireless Systems Research Group, Lucent Technologies, Bell Labs U.K., in Swindon, England. He received the Dipl.Ing. degree from the CAU Kiel in July 2001. For his diploma thesis on space-time codes he received the Prof. Dr. Werner Petersen Award. From August 2001 to October 2006 he was working toward his Ph.D. degree as a research and teaching assistant at the Information and Coding Theory Lab (ICT), Faculty of Engineering, CAU Kiel, and received his Ph.D. degree in December 2006. His research interests concern physical layer aspects of future wireless communication systems, especially multiple-input multiple-output (MIMO) systems, spatial diversity techniques, and cooperative wireless networks. He received an award from the Friends of the Faculty of Engineering for the best dissertation in 2006. Since January 2007 he is with the Communication Theory Group, Department of Electrical and Computer Engineering, University of British Columbia, in Vancouver, Canada, as a post-doctoral research fellow sponsored by the German Academic Exchange Service (DAAD).

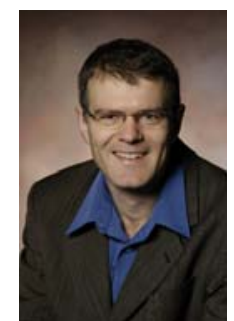

Robert Schober (M'01-SM'07) was born in Neuendettelsau, Germany, in 1971. He received the Diplom (Univ.) and the Ph.D. degrees in electrical engineering from the University of Erlangen-Nürnberg in 1997 and 2000, respectively. From May 2001 to April 2002 he was a Postdoctoral Fellow at the University of Toronto, Canada, sponsored by the German Academic Exchange Service (DAAD). Since May 2002 he has been with the University of British Columbia (UBC), Vancouver, Canada, where he is currently an Associate Professor and Canada Research Chair (Tier II) in Wireless Communications. His research interests include cooperative diversity systems, equalization, UWB, MIMO systems, and space-time processing and coding. Dr. Schober received the 2002 Heinz Maier-Leibnitz Award of the German Science Foundation (DFG), the 2004 Innovations Award of the Vodafone Foundation for Research in Mobile Communications, the 2006 UBC Killam Research Prize, and the 2007 Friedrich Wilhelm Bessel Research Award of the Alexander von Humbuldt Foundation. In addition, he received best paper awards from the German Information Technology Society (ITG), the European Association for Signal, Speech and Image Processing (EURASIP), IEEE ICUWB 2006, the International Zurich Seminar on Broadband Communications, and European Wireless 2000. Dr. Schober is also the Area Editor for Modulation and Signal Design for the IEEE TRANSACTIONS ON COMMUNICATIONS. 